\title{
Laboratory diagnosis of SARS
}

\section{A. Bermingham, P. Heinen, M. Iturriza-Gómara, J. Gray, H. Appleton and M. C. Zambon*}

\author{
Enteric, Respiratory and Neurological Laboratory, Health Protection Agency Central Public Health Laboratory, \\ 61 Colindale Avenue, London NW9 5HT, UK
}

\begin{abstract}
The emergence of new viral infections of man requires the development of robust diagnostic tests that can be applied in the differential diagnosis of acute illness, or to determine past exposure, so as to establish the true burden of disease. Since the recognition in April 2003 of the severe acute respiratory syndrome coronavirus (SARS-CoV) as the causative agent of severe acute respiratory syndrome (SARS), enormous efforts have been applied to develop molecular and serological tests for SARS which can assist rapid detection of cases, accurate diagnosis of illness and the application of control measures. International progress in the laboratory diagnosis of SARS-CoV infection during acute illness has led to internationally agreed World Health Organization criteria for the confirmation of SARS. Developments in the dissection of the human immune response to SARS indicate that serological tests on convalescent sera are essential to confirm SARS infection, given the sub-optimal predictive value of molecular detection tests performed during acute SARS illness.
\end{abstract}

Keywords: laboratory diagnosis; RT-PCR; coronavirus; immune response

\section{INTRODUCTION}

Recognition of the newly described SARS-CoV followed from its detection in clinical material from humans affected with SARS in 2003 (Drosten et al. 2003; Peiris et al. 2003a; Rota et al. 2003). The identification of the virus and its relationship to human disease were confirmed using Koch's postulates modified for viral diseases and was achieved through an international network of laboratories working under the coordination of the WHO (Kuiken et al. 2003). Experimental work has indicated that the SARS-CoV can be recovered from several organs in infected animals, indicating a disseminated infection, which parallels the observational experience of many of the laboratories involved in handling clinical samples from SARS cases (Peiris et al. 2003b). The main site of replication, pathology and recovery of the virus during human infection is considered to be the lower respiratory tract (Nicholls et al. 2003). This is consistent with the most important route of human-to-human transmission being through respiratory secretions, although outbreaks of infection that involved dissemination of virus excreted in faeces have also been described (Peiris et al. 2003b). Accurate laboratory diagnosis of SARS-CoV was essential to ensure appropriate individual patient management, local infection control and public health measures which were critical in halting the global spread of the first serious new threat to the human population in the twenty-first century.

*Author for correspondence (maria.zambon@hpa.org.uk).

One contribution of 15 to a Discussion Meeting Issue 'Emerging infections: what have we learnt from SARS?'
One of the difficulties of accurate clinical diagnosis of SARS is the relatively long incubation period after infection (mean 6-7 days but ranging up to 10-14 days), before the onset of clinical symptoms and the relatively nonspecific nature of the initial illness presentation. Early symptoms include fever, chills, non-specific malaise and myalgia compared with more florid respiratory symptoms which develop later during illness associated with pulmonary infiltrates in the lungs (Donnelly et al. 2003). Summary analyses of published case series indicate that between $25 \%$ and $75 \%$ of cases demonstrate gastrointestinal symptoms of diarrhoea, nausea and vomiting as illness progresses (Jernigan et al. 2004). The wide range of recognized gastrointestinal disturbance in different case series may be a reflection of the fact that the earliest compilations of data of the disease did not fully recognize this clinical feature. Overall, the major clinical symptoms of respiratory and enteric disease caused by the SARS-CoV in humans are analogous to disease syndromes caused by several animal coronaviruses in their natural hosts.

\section{CLINICAL VIROLOGY}

Clinical studies on SARS have shed light on the diagnostic usefulness of different samples at different times during illness, summarized in figure 1 . In many viral illnesses, virus shedding is greatest during the early symptomatic phase of illness around the onset of symptoms, e.g influenza (Hayden et al. 1998). However, with SARS$\mathrm{CoV}$, virus excretion is comparatively low during the initial phase of illness and it is necessary to use very sensitive tests that are able to detect low levels of viral nucleic acid during the first days of illness. The mainstay of diagnosis during the illness phase of SARS has involved the use of RT-PCR to detect the SARS-CoV nucleic acid 


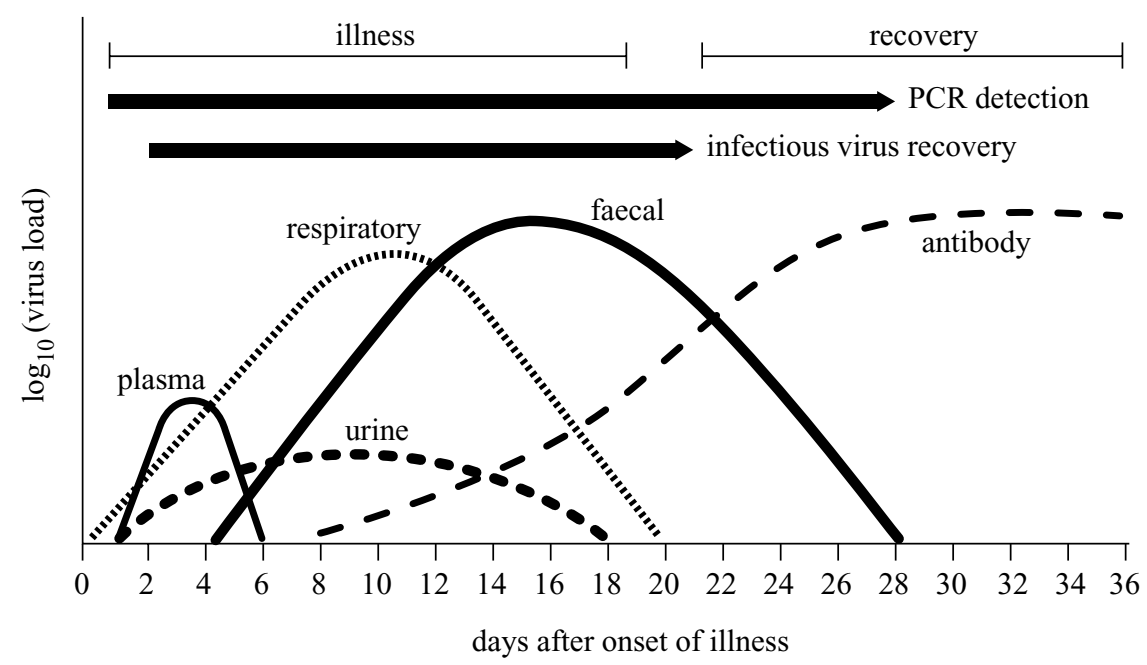

Figure 1. Schematic diagram of the course of virus shedding and detection in body fluids during SARS illness and recovery. Onset of illness is taken to be the onset of symptomatic fever.

amplified directly from clinical samples. RT-PCR protocols were developed with unprecedented speed as a result of the efforts of the WHO collaborative laboratory network. Samples of different body fluids such as blood, respiratory secretions, urine, stool and lung tissue from suspected and probable cases of SARS were analysed. The end point of detection for the SARS-CoV was similar to that found in previously described protocols for detection of known human coronaviruses (Vabret et al. 2001).

It is clear that viral load increases in respiratory samples in the second week of SARS illness (Peiris et al. 2003b), and that the viral load is greatest in samples taken from lower in the respiratory tract (Drosten et al. 2003), peaking around day 10, with the peak of viral detection in faeces coming slightly later. SARS-CoV RNA was detected in only $32 \%$ of individuals in nasopharyngeal aspirates at initial presentation (mean 3.2 days after illness onset), but in $68 \%$ at day 14 (Peiris et al. 2003b), and in over $90 \%$ of faecal samples collected in the second week of illness, peaking around days 15-17 (Chan et al. 2004). Quantification indicated that viral load in respiratory secretions peaked at day 10 with a geometric mean titre of $1.9 \times 10^{7}$ copies $\mathrm{ml}^{-1}$. The clinical features of SARS illness therefore appear to be a good reflection of the body compartments/fluids in which SARS virus has been detected or recovered, with a clear time course. The detection of virus replication in different body compartments over several weeks, before resolution or progression to death, underlies the suggested use of different clinical samples to detect virus at different times after illness onset (figure 1). More recently, analysis of sequential samples of plasma from patients with SARS during early illness using PCR indicates that there is an early peak of viraemia, with up to $70 \%$ of samples containing detectable virus in the first few days after onset of illness (figure 2; Grant et al. 2003). This suggests that a viraemic phase is then most probably followed by increasing virus replication in the lower airways and gastrointestinal tract. Taken together, these observations indicate that sampling to detect SARS$\mathrm{CoV}$ in the first week after onset of illness should involve the simultaneous collection and analysis of different clinical samples, including respiratory samples from as low in the respiratory tract as is practicable, blood, faeces and

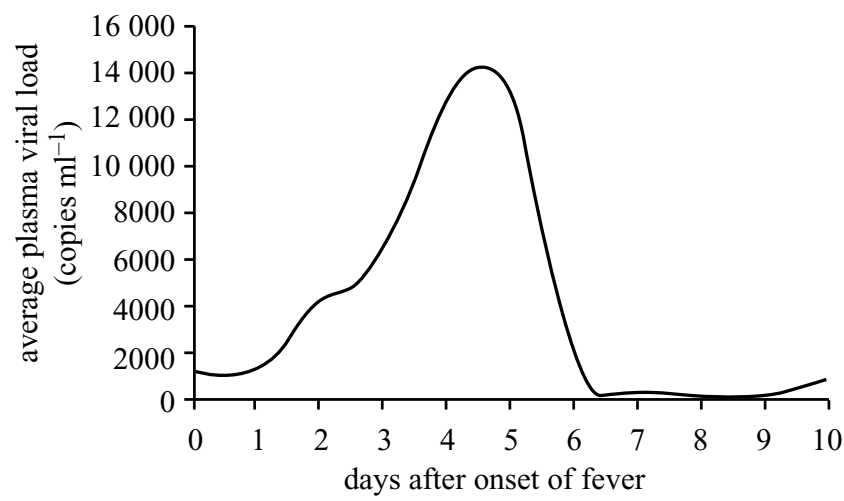

Figure 2. Schematic diagram of the detection of SARS viral RNA using RT-PCR in plasma in early SARS illness. Compiled from Grant et al. (2003).

urine. Detection of virus in the second week after the onset of illness is actually more likely, given the higher viral load, and should also involve sampling from multiple sites.

\section{MOLECULAR DETECTION}

Despite reasonably high rates of detection of virus in clinical samples, and good analytical sensitivity of the tests themselves, the predictive value of molecular diagnostic tests in the early stages of illness are still sub-optimal as they cannot rule out the presence of SARS-CoV. This is partly a reflection of the variable viral load in clinical samples, particularly in respiratory samples, which are most likely to be taken from the upper respiratory tract where the priority is to minimize aerosol generation when sampling to prevent infection of healthcare workers. This and the fact that viral replication does not appear to peak until some time after the onset of disease may result in sub-optimal samples. Obtaining a clear diagnosis may be difficult when the disease symptoms are least specific.

Parallel testing of samples for other infectious agents such as influenza, Mycoplasma pneumoniae, Legionella pneumophila and human metapneumovirus, which are capable of causing a similar clinical syndrome, is essential in the differential diagnosis early after disease onset and 
may help to exclude SARS, particularly in returning travellers from countries where SARS is considered likely to re-emerge from an animal reservoir, although co-infection of SARS with other respiratory pathogens can occur (Poutanen et al. 2003). If the presence of an alternative diagnosis is to be used as the justification for discontinuing SARS-specific isolation procedures, the diagnosis should be based on tests with a high predictive value and the clinical illness should be fully explicable by the alternative diagnosis. Testing of multiple sequential samples increases the reliability of laboratory diagnosis, and reduces the likelihood of false-positive results, which is always of concern when using sensitive molecular diagnostic techniques. These findings underlie the current stringent WHO recommendations about the confirmation and quality control of SARS laboratory diagnosis: 'laboratories performing SARS specific PCR tests should adopt strict criteria for confirmation of positive results, especially in low prevalence areas where the predictive values might be lower' (Galen \& Gambino 1975). This guidance includes the current requirement for detection of virus by RT-PCR in two different samples (e.g. respiratory and faecal), or sequential samples from the same body site on different days and robust confirmatory strategies. Examples of laboratory-acquired infection that occurred in Southeast Asia in 2003-2004, leading to extensive deployment of healthcare resources for contact tracing and quarantine, emphasize the necessity of stringent biosafety considerations in laboratories diagnosing SARS.

\section{VIRUS TARGETS FOR DIAGNOSIS}

Initial diagnostic work focused on the molecular detection of SARS-CoV RdRp $(P o l)$ gene, because the $P o l$ gene sequences were the first available (Drosten et al. 2003), and the $\mathrm{Pol}$ region of the coronavirus genome is well conserved across all coronaviruses. The use of detection probes involving degenerate primer sets that can detect all known coronaviruses (Stephensen et al. 1999) remains a useful screening approach, because this allows the deployment of a pan-corona molecular strategy, which will detect all known human coronaviruses, some of which may possibly cause diseases that overlap with the clinical syndrome of SARS. This approach can be run in parallel with RTPCRs which are absolutely specific for SARS-CoV (Yam et al. 2003). The sequence conservation in the Pol region across all coronaviruses is such that diagnostic SARS-CoV tests based on the $P o l$ region of the genome should, as part of a validation process, exclude detection of $229 \mathrm{E}$ (group 1 coronaviruses) and OC43 (group 2 coronaviruses) to avoid false-positive detections of human coronaviruses.

Coronaviruses have unusually long RNA genomes of $c a$. 30000 bases. Viral replication and transcription is complex. As data have developed about the nature of SARS$\mathrm{CoV}$ replication in vitro, it is evident that there is a transcription gradient across the viral genome in common with other coronaviruses (Thiel et al. 2003). The genomic organization of the coronavirus, with the non-structural genes at the $5^{\prime}$ end and structural genes at the $3^{\prime}$ end, reflects this transcription strategy. Differential transcription generates a gradient of nested sgRNAs sharing a common $3^{\prime}$ end. Genes encoded at the $3^{\prime}$ end are transcribed at high levels and represent the most abundant sgRNA species at least during infection in cell culture. Products of non-structural genes, such as the $\operatorname{RdRp}(P o l)$, responsible for replication and transcription of the viral genome, are needed in smaller amounts than structural genes, such as the NC protein involved in assembly of the virions. Consequently, sgRNA for the NC gene should be more abundant than sgRNA for the Pol gene in infected cells. This feature of coronavirus biology may be relevant for improving its detection in clinical practice. There are considerable differences in the concentration of viral RNA fragments in infected cells, with several log-fold increases in the amount of NC (mRNA) RNA in infected cells, compared with the transcripts of $\mathrm{Pol}$ genes (Thiel et al. 2003). This finding suggests that there may be some diagnostic advantages to targeting NC genes for molecular detection as well as other genes, to improve the overall sensitivity of detection, because the amount of viral template will be much higher, if clinical material contains virus infected cells as well as whole virus. Using clinical samples spiked with a mixture of SARS-CoV virus and infected cells, it is evident that detection of NC genes does provide some additional sensitivity (table 1). Several laboratories have developed diagnostic PCRs for the detection of other regions of the genome, particularly the viral NC gene (Emery et al. 2004). This approach is consistent with the observation that targeting the SARS-CoV NC region improved the sensitivity of detection more than a 100-fold in experimentally infected animals (Kuiken et al. 2003).

\section{DIAGNOSTIC DEVELOPMENTS}

As might be expected in the first several months after the emergence of a new human pathogen, there has been an explosion of diagnostic developments, particularly in the commercial sector. One of the difficulties in validating new diagnostic tests is the availability of clinical material because over $90 \%$ of the cases worldwide occurred in Southeast Asia. It is likely that incremental gains in sensitivity of SARS-CoV PCR tests will occur over the next few years as there is increasing use of real-time PCR platforms capable of detecting multiple targets and concurrent or multiplexing of SARS-specific and pan-corona tests. Greater gains in sensitivity may also come from techniques that concentrate the biological sample before processing for nucleic acid extraction (Grant et al. 2003; Chan et al. 2004).

\section{VIRUS PROPAGATION}

It is fortunate that the SARS-CoV virus, in contrast to many animal coronaviruses, can be cultured easily in a variety of continuous cell lines, including FRhK and Vero E6 cells, produces a recognizable and distinct widespread $\mathrm{CPE}$ (figure 3), and grows well at $33^{\circ} \mathrm{C}$ and $37^{\circ} \mathrm{C}$. This has allowed the recovery of infectious virus from affected individuals, which in turn expedited sequencing of the entire virus genome (Marra et al. 2003). Moreover, the development of infectivity assays has allowed quantification of virus infectivity and development of neutralization assays (plaque reduction neutralization tests are shown in figure 4). Infectious virus has not been recovered beyond three weeks after illness onset even though virus 


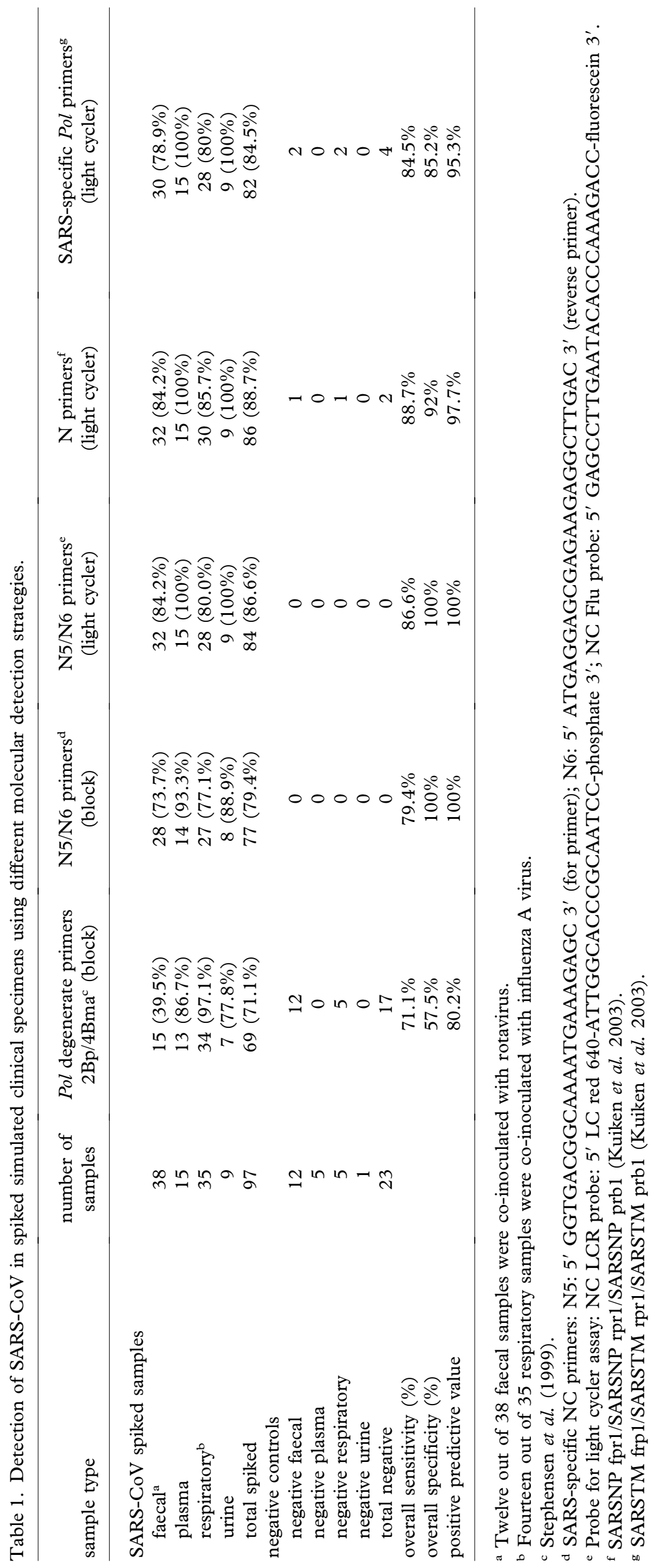


(a)

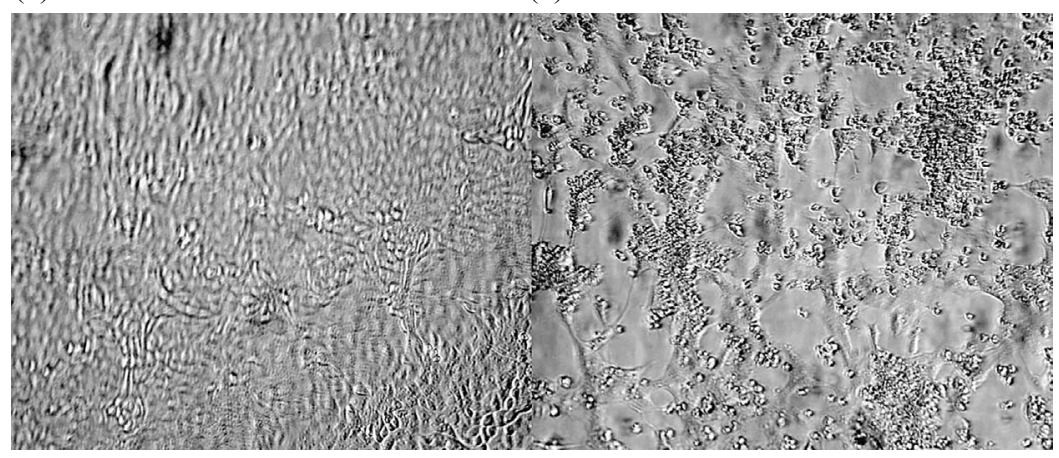

Figure 3. Mock infected Vero E6 cells (a) compared with CPE of SARS in Vero E6 cells (b) at 4 days after infection.

(a)

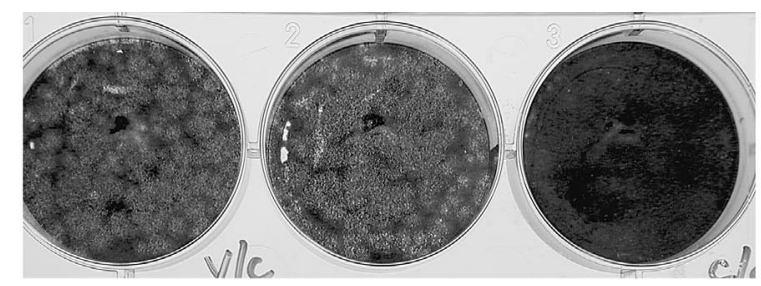

(b)

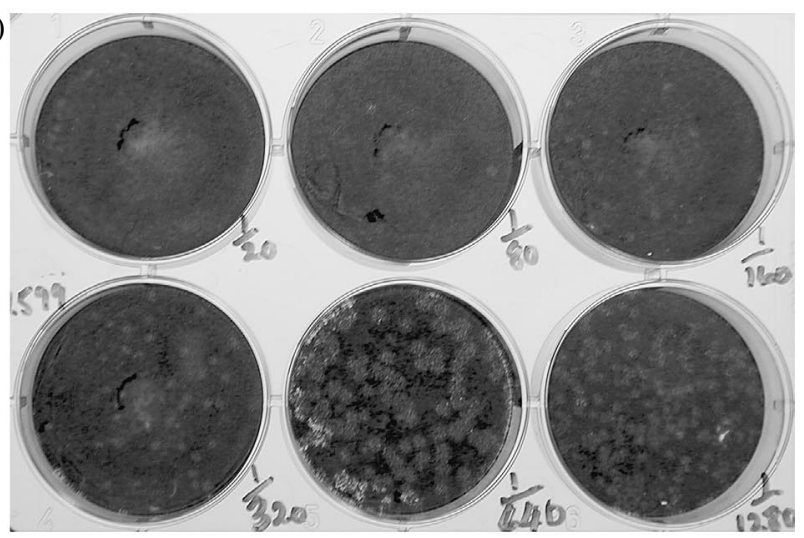

(c)

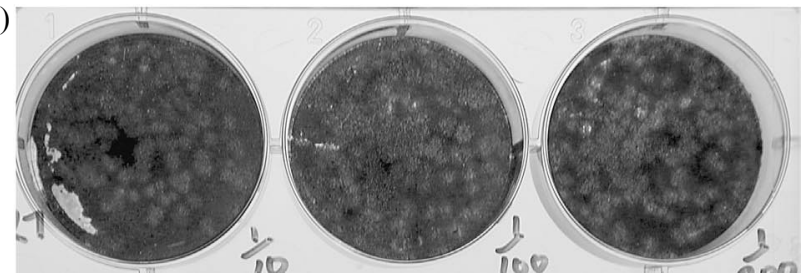

Figure 4. Neutralizing antibody tests. Infectivity $(a)$ and plaque reduction assays $(b, c)$ for the detection of SARSneutralizing antibodies. Virus-infected cells are shown in the first two wells of $(a)$, indicating clear plaque formation in Vero E6 cells 4 days after inoculation, with mock infected cell control in the third well. Infection of virus in the presence of increasing dilution of SARS convalescent serum is shown in $(b)$ and indicates total inhibition of virus growth at day 4 after inoculation with an $\mathrm{IC}_{50}$ serum titre of $c a .1$ in 320. Non-SARS serum with no neutralizing activity is shown in $(c)$.

is still detectable by RT-PCR for several days or weeks after this. The inability to detect infectious virus indicates the natural cessation of viral replication or the development of the antibody response (detectable from about day 10 after onset of illness) which may form complexes with virus, thereby affecting the ability to detect infectious particles.

\section{SEROLOGICAL ASSAYS}

As the virus produced a CPE in FRhK cells, virusinfected cells were used as an antigen substrate before the aetiological agent had been described. Seroconversion using IF was the earliest serological test used to detect SARS (Peiris et al. 2003a). Use of IF indicated that seroconversion took place $c a$. 10 days after illness onset, but might not actually be evident in all cases until ca. 28 days after onset, as ca. $10-20 \%$ of individuals did not have detectable antibodies until after day 21 (Peiris et al. $2003 b$ ). The late seroconversions noted may reflect the fact that many patients were treated with high-dose steroids, which is likely to have delayed the antibody response, although this cannot be firmly concluded from the clinical datasets available. The only available laboratory method for excluding the diagnosis of SARS-CoV infection is to obtain a negative result on serological testing of a convalescent phase serum at 28 days after onset of symptoms. It is therefore essential for understanding the true disease burden to have robust and reliable serological tests.

The development of SARS ELISA tests followed rapidly after the identification of the virus, and the use of virally infected cells to prepare antigen for indirect ELISAs for the detection of SARS antibodies ( $\operatorname{IgM}$ and $\operatorname{IgG}$ ) has been possible because of the ability to culture virus to reasonable titre, and to use virus-infected cells as a source of antigens (figure 5; Ksiazek et al. 2003; Kuiken et al. 2003).

Analysis of antibody responses to SARS-CoV has so far shown limited cross reactivity with antibodies to human group 1 or group 2 coronaviruses. Although full evaluations are not complete yet and further data are required, some limited cross reactivity to group 1 animal coronaviruses has been noted (Ksiazek et al. 2003). The method of preparation of antigens and the formulation of serological tests may impact substantially on the ability to detect any cross-reacting antibody. Understanding the impact of serological responses to other human coronaviruses on antibody response to SARS-CoV is important because it will affect the specificity of tests and conclusions about exposure to SARS-CoV in the absence of clinical illness. Serological data are developing rapidly and early data suggest that high levels of neutralizing antibodies are formed after SARS infection and last for at least several months after infection. The use of neutralizing antibody tests (such as shown in figure 4) indicates that antibody to SARS may also cross neutralize related animal viruses, perhaps with a slightly lower titre, and this is taken as a 
(a)

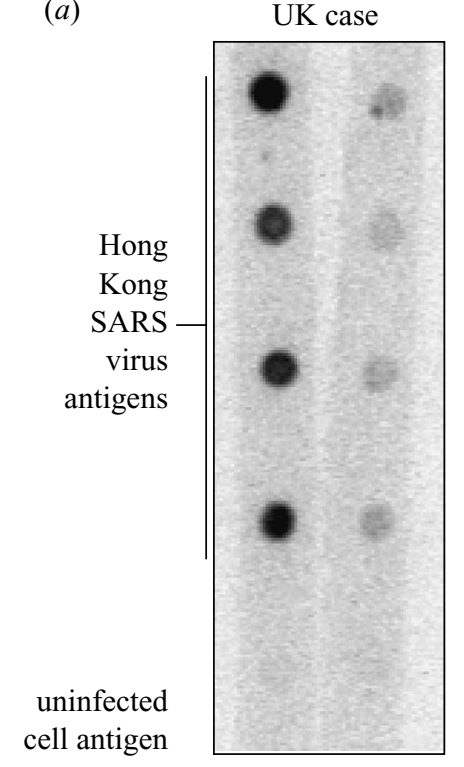

(ii) (i) (b)

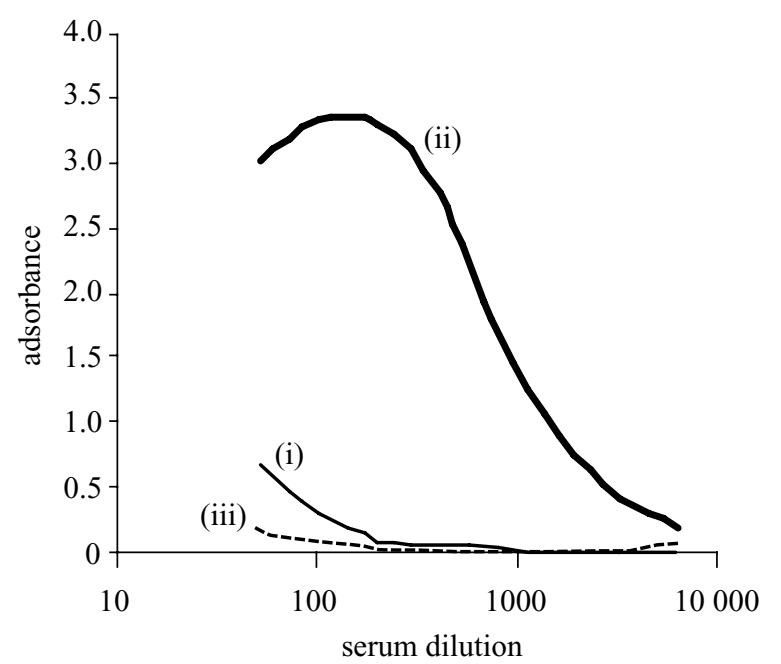

28 days

Figure 5. Detection of antibodies to SARS-infected cell antigen by dot blot (a)(i,ii) and ELISA $(b)$. (a) Sera from SARSpositive UK probable case (Kuiken et al. 2003) at (i) day 7 acute, and (ii) day 28 convalescent, is shown in dot blot assay formats indicating the detection of low levels of antibody early after illness onset and seroconversion at day 28 . (b) ELISA reactivity of serial dilutions of serum taken from: (i) acute SARS day 7; (ii) day 28 convalescent SARS; and (iii) day 28 after influenza A illness presenting with a clinical syndrome fulfilling the WHO case definition for 'probable SARS'.

suggestion of more than one serogroup of SARS-CoV (Zheng et al. 2004).

The specificity of the SARS-CoV antibody response has allowed seroprevalence studies to be undertaken using IF, which have concluded that there was little spread of SARS-CoV in the general population in Hong Kong, based on blood donor screening (Donnelly et al. 2003). However, up to $40 \%$ of humans who are market traders of live animals or who are restaurant workers preparing exotic meat of the putative wild animal reservoir (members of the vivverid, mustelid and canid families), showed evidence of exposure to SARS viruses, which has been taken to support the zoonotic origin of the SARSCoV (Guan et al. 2003). Screening of archived healthy adult sera in Hong Kong (Zheng et al. 2004) taken before the SARS outbreak indicated that a few had detectable antibody to the SARS-CoV, suggesting pre-existing evidence of exposure to a related virus.

One of the difficulties of screening individual sera, whether from cases of illness, for serosurveillance or for contact tracing, is the sensitivity and specificity of ELISAs or IF assays, which typically have sensitivities and specificities between $90 \%$ and $98 \%$. This is true for almost all ELISAs used to screen human sera for many viral diseases, and usually leads to an algorithm of a screening assay followed by a confirmatory assay. Invariably, a small proportion of reactive sera will not be true positives after the first ELISAs. To improve the certainty of diagnosis, a serological strategy needs to be adopted, involving a second tier of tests (figure 6 ) with or without an additional second serum to test for seroconversion. Many laboratories have adopted a neutralization test as a 'gold standard' confirmatory assay, with typical neutralizing antibody titres of between several hundred and several

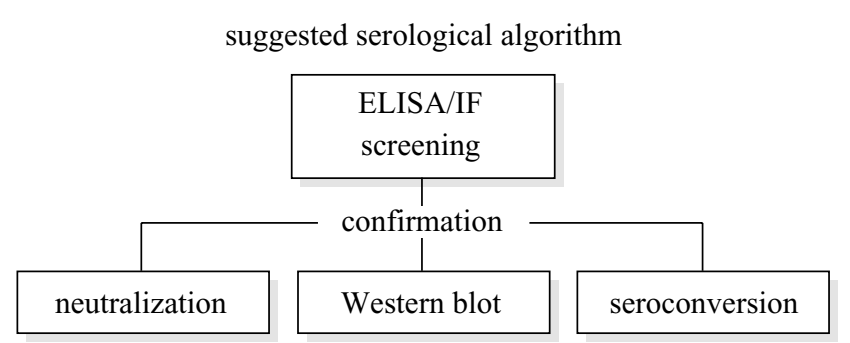

Figure 6. Suggested algorithm for serological testing of SARS.

thousand detectable at 28 days after onset of illness. The rise in neutralizing antibody may not exactly parallel the rise in total antibody detection, and may be somewhat slower to develop. However, tests that use virus-infected cells, or live virus, as required for whole-cell lysate ELISA assays, IF or neutralization tests, require the growth of virus (figures 4 and 5), which in turn requires a biosafety level 3 laboratory and prevents the tests being used widely. It is likely that trends in serological assay development will be towards the use of recombinant antigen ELISAs and finding surrogate methods for neutralization tests such as receptor binding assays, which may be a safer alternative for the serological diagnosis of SARS. The limited data available internationally so far suggest good correlation between recombinant protein ELISAs, Western blots and IF results (Wu et al. 2004), but much more evaluation will be required to fully understand the relationships between antibodies to different coronaviruses.

There remain many unanswered questions about the nature of serological responses to infection with the SARS-CoV, despite the astonishing rapidity of development of robust diagnostic tests. The next few years will 
undoubtedly see the unravelling of the relationship between neutralizing and functional antibody and total antibodies to specific virus proteins, the duration and magnitude of antibody response in the context of disease protection and a comparison of antibody response in children and adults. An attempt to understand serological relationships between different SARS-CoV viruses as well as between SARS-CoV and other human and non-human coronaviruses will benefit our understanding of the biology of coronaviruses as a whole, and assist understanding of the severity of SARS disease in humans. A very significant side effect of SARS-related research is likely to be much more focus on the burden of illness as a result of human coronaviruses and their role in acute respiratory and gastrointestinal infections, a neglected backwater of human virology.

The authors acknowledge the excellent technical work of the following members of the Health Protection Agency ERNVL laboratory staff: Ruth Reith, Jo Smith, Tracey Kesting, Ian Putwain, Nelita du Plessis, Victoria Gould, Carol Sadler and Brian Megson who assisted in the development of diagnostic tests for SARS in the UK, and the support and advice of colleagues including Dr Robin Gopal, Dr Dhan Samuels, Dr Joanna Ellis, Dr Joanne Stockton, Pam Litton, Judy Mitchell and Dr Kirstin Edwards.

\section{REFERENCES}

Chan, K. H., Poon, L. L. L. M., Cheng, V. C. C., Guan, Y., Hung, I. F. N., Kong, J., Yam, L. Y. C., Seto, W. H., Yuen, K. Y. \& Peiris, J. S. M. 2004 Detection of SARS coronavirus in patients with suspected SARS. Emerg. Infect. Dis. 10, 294-299.

Donnelly, C. (and 19 others) 2003 Epidemiological determinants of spread of causal agent of severe acute respiratory syndrome in Hong Kong. Lancet 361, 1761-1766b.

Drosten, C. (and 25 others) 2003 Identification of a novel coronavirus in patients with severe acute respiratory syndrome. New Engl. f. Med. 348, 1967-1976.

Emery, S. L. (and 15 others) 2004 Real-time reverse transcription-polymerase chain reaction assay for SARS-associated coronavirus. Emerg. Infect. Dis. 10, 311-316.

Galen, R. S. \& Gamhino, S. R. 1975 Beyond normality: the predictive value and efficiency of medical diagnosis. New York: Wiley.

Grant, P. R., Garson, J. A., Tedder, R. S., Chan, P. K., Tam, J. S. \& Sung, J. J. 2003 Detection of SARS coronavirus in plasma by real-time RT-PCR. New Engl. f. Med. 349, 2468-2469.

Guan, Y. (and 17 others) 2003 Isolation and characterization of viruses related to the SARS coronavirus from animals in southern China. Science 302, 276-278.

Hayden, F. G., Fritz, R., Lobo, M. C., Alvord, W., Strober, W. \& Straus, S. E. 1998 Local and systemic cytokine responses during experimental human influenza A virus infection. Relation to symptom formation and host defense. F. Clin. Invest. 101, 643-649.

Jernigan, J., Low, D. E. \& Helford, R. F. 2004 Combining clinical and epidemiological features for early recognition of SARS. Emerg. Infect. Dis. 10, 327-333.
Ksiazek, T. G. (and 26 others) 2003 A novel coronavirus associated with severe acute respiratory syndrome. New Engl. $\mathcal{F}$. Med. 348, 1953-1966.

Kuiken, T. (and 20 others) 2003 Newly discovered coronavirus as the primary cause of severe acute respiratory syndrome. Lancet 362, 263-269.

Marra, M. A. (and 59 others) 2003 The genome sequences of the SARS associated corona virus. Science 300, 1399-1404.

Nicholls, J. M. (and 15 others) 2003 Lung pathology of fatal severe acute respiratory syndrome. Lancet 361, 1773-1778.

Peiris, J. S. M. (and 16 others) $2003 a$ Coronavirus as a possible cause of severe acute respiratory syndrome. Lancet 361, 1319-1325.

Peiris, J. S. M. (and 17 others) $2003 b$ Prospective study of the clinical progression and viral load of SARS-associated coronavirus pneumonia in a community outbreak. Lancet 361, 1767-1772.

Poutanen, S. M. (and 19 others) 2003 Identification of severe acute respiratory syndrome in Canada. New Engl. f. Med. 348, 1995-2005.

Rota, P. A. (and 34 others) 2003 Charaterization of a novel coronavirus associated with severe acute respiratory syndrome. Science 300, 1394-1399.

Stephensen, C. B., Casebolt, D. B. \& Gangopadhyay, N. N. 1999 Phylogenetic analysis of a highly conserved region of the polymerase gene from 11 coronaviruses and development of a consensus polymerase chain reaction assay. Virus Res. 60, 181-189.

Thiel, V. (and 11 others) 2003 Mechanisms and enzymes involved in SARS coronavirus genome expression. F. Gen. Virol. 84, 2305-2315.

Vabret, A. F., Mouthon, T., Mourez, S., Gourain, J., Petitjean, J. \& Freymouth, F. 2001 Direct diagnosis of human respiratory coronaviruses $229 \mathrm{E}$ and OC43 by the polymerase chain reaction. F. Virol. Meth. 97, 59-66.

Wu, H. S. (and 17 others) 2004 Serologic and molecular biologic methods for SARS associated coronavirus infection: Taiwan. Emerg. Infect. Dis. 10, 304-310.

Yam, W. C., Chan, K. H., Poon, L. L. M., Guan, Y., Yuen, K. Y., Seto, W. H. \& Peiris, J. S. M. 2003 Evaluation of reverse transcription-PCR assays for rapid diagnosis of severe acute respiratory syndrome associated with a novel coronavirus. F. Clin. Microbiol. 41, 4521-4524.

Zheng, B. J., Guan, Y., Wong, K. H., Zhou, J., Wong, K. L., Young, B. W. Y., Lu, L. W. \& Lee, S. S. 2004 SARS related virus predating SARS outbreak, Hong Kong. Emerg. Infect. Dis. 10, 176-178.

\section{GLOSSARY}

CPE: cytopathic effect

ELISA: enzyme linked immunosorbent assay

IF: immunofluorescence

NC: nucleocapsid

$\operatorname{RdRp}(\mathrm{Pol})$ : RNA-dependent RNA polymerase

RT-PCR: reverse transcription-polymerase chain reaction

SARS: severe acute respiratory syndrome

SARS-CoV: severe acute respiratory syndrome coronavirus

sgRNA: sub-genomic RNA

WHO: World Health Organization 